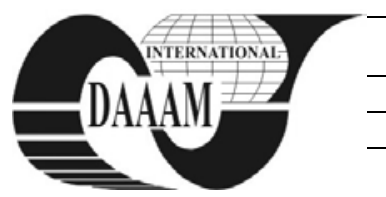

\title{
NOTIONAL DECISION ANALYSIS AND RESOLUTION ON PRODUCTION MANAGEMENT SYSTEMS
}

\author{
FILIP, F[lorina] - C[ristina] \& SHIRVANI, H[assan]
}

\begin{abstract}
The main objectives of this study (decision process) is to identify and select alternatives based on established criteria that results in reduction of the subjectivity decision. Historically, criteria and rationales for previous decisions are documented and traceable consequently they can be used to support similar decision. This paper uses the description process tool "decision analysis and resolution" concerning a case study at organisation level, and explains the target and handling of the decision process.

Key words: process, decision, analysys, evaluation
\end{abstract}

\section{INTRODUCTION}

Decision process is the study of identifying and choosing alternatives based on the values and preferences of the decision maker. Making a decision implies that there are alternative choices to be considered, and in such cases, not only we want to identify as many of these alternatives as possible but, to choose the one that has the highest probability of success or effectiveness and best fits our goals, desires, lifestyle and values. Now a day's very few decisions are made with absolute certainty because complete knowledge about all the alternatives is seldom possible. Thus, every decision involves a certain amount of risk. If there is no uncertainty, no decision is normally necessary, hence a set of algorithm, steps or a recipe that is followed to bring about a fixed desirable result.

An ideal decision environment would include all the information, and every possible alternative. Information and alternatives are constrains because the time and effort to gain information or identify alternatives are limited. The time constraint simply means that a decision must be made by a certain time. The effort constraint reflects the limits of manpower, cost, and priorities (Harris, 2009).

Decision analysis is the discipline of evaluating complex alternatives in terms of values and uncertainty. Values are generally expressed economically because this is a major concern for management. Furthermore, decision analysis provides insight into how the defined alternatives differ from one another and then generates suggestions for new and improved alternatives.

A decision needs a decision maker who is responsible and accountable for making the decisions. Normally, the decision maker has a number of alternatives and must choose one of them. The objective of the decision-maker is to choose the best alternative. Once a decision has been made, events that the decision-maker has no control over may have occurred. Each combination of alternatives, followed by an event happening, leads to an outcome with some measurable value. Managers are expected to make decisions in complex situations (Arsham H.).

\section{DECISIONAL PROCESS DESCRIPTION}

\subsection{Process Roles and Stakeholders}

The stakeholders are relevant people like staff, customers and suppliers, whom they have a special interest in the results of a process and may be affected by the results they may also be involved in the development of a process.

The roles are generic roles which are mapped to process roles and are dependent on the issue of the decision (e.g. decision client $=$ project manager, decision manager $=$ technical architect/tool expert and decider $=$ senior manager).

Decision client is the ordering customer who initiates a formal decision process. The decision client can be any authority in the organisation that has to obtain a solution based on a formal decision. Responsibilities: obtains a thorough understanding of the decision issue as a basis for applying the process, provides the necessary resources to establish a decision and identifies the suitable decision maker.

Decision manager is the operating manager of the formal decision process. Responsibilities: creates and controls an adequate plan comprising effort, tasks, schedule and resources (typically a part of the project plan), establishes evaluation criteria, identifies and evaluates alternative solutions, selects evaluation methods and a recommended solution.

Decider makes the decision and is accountable for the consequences.

\subsection{Inputs and Outputs Process, Entry and Exit Criteria}

Inputs process include the decision issue (the issue that has to be decided upon) and the local decision strategy (including guidelines when to apply this process, typical selection criteria and typical evaluation methods). Outputs process includes the approved solution.

Entry criteria processes are needed for decision identified. Exit criteria process includes the decision data that are archived for retrieval.

\section{DECISIONAL PROCESS ACTIVITIES}

\subsection{Initiate the Decision Process}

- Entry criteria: need for applying a formal evaluation process identified.

- Inputs: decision issue.

- Activity steps: obtain a thorough understanding of the decision issue, identify the appropriate decider for the decision issue, and assign a decision manager.

- Responsible: decision client.

- Stakeholder (involvement): decision manager (decider).

- Exit criteria: decision issue is defined, decider and decision manager are assigned.

\subsection{Prepare Evaluation}

- Entry criteria: decision issue is defined, decider and decision manager are assigned.

- Inputs: decision issue, decision process constraints (e.g. resources, budget, schedule, legal).

- Activity steps: create and control an adequate plan comprising effort, tasks, schedule and resources (typically a part of the project plan), select and adapt the evaluation criteria for alternatives, select evaluation method, identify, 
collect or generate alternative solutions, consider previous similar decisions (if available).

- Responsible: decision manager.

- Stakeholder (and involvement): decision client(s), engineer(s).

- Outputs: evaluation criteria, alternative solutions, evaluations method(s), planning.

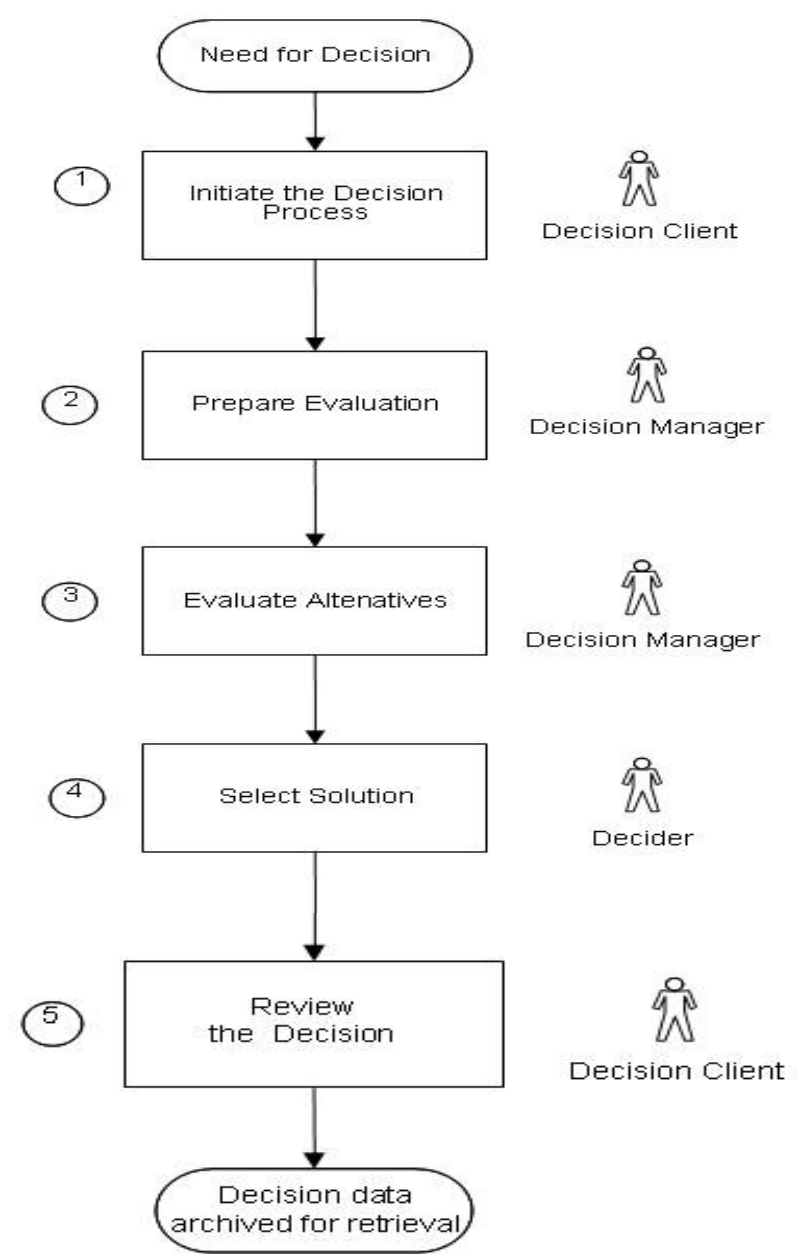

Fig. 1. Decisional process flowchart

\subsection{Evaluate Alternatives}

- Inputs: evaluation criteria, alternative solutions, evaluation methods.

- Activity steps: evaluate the alternatives applying the selected evaluation methods and evaluation criteria, select a recommended solution, document rated alternatives and recommended solution.

- Templates/checklists: site-specific decision matrix.

- Responsible: decision manager.

- Stakeholder (and involvement): engineer(s).

- Outputs: rated alternative solutions, recommended solution.

\subsection{Select Solution}

- Inputs: rated alternative solutions, recommended solution.

- Activity steps: make a decision (select the solution out of the rated alternatives taking into account the adhering risks), capture rationale for the decision.

- Templates/checklists: site-specific decision matrix.

- Responsible: decider.

- Stakeholder (and involvement): decision client(s), decision manager(s).

- Outputs: approved solution.

- Exit criteria: decision has been made.

\subsection{Review the Decision}

- Entry criteria: decision has been made.

- Inputs: decision issue.

- Approved solution: evaluation methods, alternative solutions, evaluation criteria, decision matrix.

- Activity steps: archive consolidated documents to support retrieval of decision, perform lessons learned (if applicable), review the decision afterwards to gain input for the improvement of evaluation methods and criteria.

- Templates/checklists: lessons learned template (if applicable).

- Responsible: decision client

- Stakeholder (and involvement): decision manager(s).

- Outputs: archived data, lessons learned (if applicable), mprovement requests concerning evaluation methods and criteria.

- Exit criteria: decision data is archived for retrieval.

\section{CONCLUSION}

The proposed decision process can be applied whenever important decisions have to be taken. It provides guidance for technical, management and all other decisions that have a significant impact on the business unit or project. It should not be used for unimportant decisions such as buying office supplies. Criteria and rules when to apply the decision processes are provided by the site-specific decision rules. If a formal decision process is recommended or mandatory in the context of an existing process, then this is used to provide process specific rules, templates and criteria.

The main goal of the decision process is to reduce the bias of subjectivity and the risk within decisions by taking important decisions in a systematic manner. The decision process is applicable to various types of decisions and organizational units.

\section{ACKNOWLEDGEMENTS}

This paper is supported by the Sectoral Operational Programme Human Resources Development (SOP HRD), financed from the European Social Fund and by the Romanian Government under the contract number POSDRU/88/1.5/S/59321.

\section{REFERENCES}

Arruda, E.F. \& Fragoso, M.D. (2011). Time aggregated Markov decision processes via standard dynamic programming. Operations Research Letters, Vol. 39, No. 3, (May 2011), pp. 193-197

Arsham, H. Tools for Decision Analysis: Analysis of Risky Decisions, Available from: http://home.ubalt.edu/ntsbarsh/opre640a/partix.htm Accessed on: 2011-06-27

Ullman, D.G. (2006). Making Robust Decisions: Decision Management for Technical, Business, and Service Teams, Trafford Publishing, ISBN 978-1425109561, UK

Forman, E.H. \& Selly, M.A. (2001). Decision by objectives: how to convinge others that you are right, World Scientific Publishing, ISBN 981-02-4142-9, Singapore

Riedl, R.; Brandstätter, E. \& Roithmayr, F. (2008). Identifying decision strategies: A process and outcome based classification method. Behavior Research Methods, Vol. 40, No 3, pp. 795-807, ISSN: 1554-3528

Harris, R. (2009). Introduction to Decision Making, Available from: http://www.virtualsalt.com/crebook5.htm Accessed: 2011-06-28 